\title{
Robotics for Advanced Therapeutic Colonoscopy
}

\author{
Jennie Y Y Wong and Khek Yu Ho \\ Department of Medicine, Yong Loo Lin School of Medicine, National University of Singapore, Singapore
}

\begin{abstract}
Although colonoscopy was originally a diagnostic imaging procedure, it has now expanded to include an increasing range of therapeutic interventions. These procedures require precise maneuvers of instruments, execution of force, efficient transmission of force from the operator to the point of application, and sufficient dexterity in the mobilization of endoscopic surgical instruments. The conventional endoscope is not designed to support technically demanding endoscopic procedures. In case of colonoscopy, the tortuous anatomy of the colon makes inserting, moving, and orientating the endoscope difficult. Exerting excessive pressure can cause looping of the endoscope, pain to the patient, and even perforation of the colon. To mitigate the technical constraints, numerous technically enhanced systems have been developed to enable better control of instruments and precise delivery of force in the execution of surgical tasks such as apposing, grasping, traction, counter-traction, and cutting of tissues. Among the recent developments are highly dexterous robotic master and slave systems, computer-assisted or robotically enhanced conventional endoscopes, and autonomously driven locomotion devices that can effortlessly traverse the colon. Developments in endoscopic instrumentations have overcome technical barriers and opened new horizons for further advancements in therapeutic interventions. This review describes examples of some of these systems in the context of their applications to advanced therapeutic colonoscopy. Clin Endosc 2018;51:552-557
\end{abstract}

Key Words: Robotics; Flexible endoscopy; Colonoscopy; Therapeutic endoscopy

\section{INTRODUCTION}

While colonoscopy remains primarily a diagnostic imaging modality, a substantial proportion of currently performed colonoscopy procedures are interventional in nature. These therapeutic interventions may include endoscopic submucosal dissection of preneoplastic or early malignant colorectal lesions. These intricate procedures are highly demanding in terms of the skill of the endoscopist. Typically, specialized training and a certain amount of experience are required to achieve competency in endoscopy. ${ }^{1}$ A series of recent studies showed a perforation rate of $5.5 \%-10 \%$ in endoscopic sub-

Received: May 31, 2018 Revised: July 3, 2018

Accepted: July 7, 2018

Correspondence: Khek Yu Ho

Department of Medicine, Yong Loo Lin School of Medicine, National University of Singapore, NUHS Tower Block, 1E Kent Ridge Road Level 11, 119228, Singapore

Tel: +65-6772-4362, Fax: +65-6779-4112, E-mail: mdchoky@nus.edu.sg ORCID: https://orcid.org/0000-0002-2932-1962

(cc) This is an Open Access article distributed under the terms of the Creative Commons Attribution Non-Commercial License (http://creativecommons.org/ licenses/by-nc/3.0) which permits unrestricted non-commercial use, distribution, and reproduction in any medium, provided the original work is properly cited. mucosal dissection of colorectal tumors. ${ }^{2-5}$ The endoscopist must, therefore, also possess the skills to manage inadvertent perforation and bleeding. Most therapeutic interventions require sufficient dexterity in the mobility of endoscopically deployed instruments and precise execution of force at the point of application. Despite the availability of a wide range of advanced ancillary devices (suturing, hemostatic, and traction devices) and interchangeable endoscopic accessories such as needles, electrosurgical knifes, graspers, forceps, endoclips, and electrocoagulation probes, standard endoscopes used in mainstream practices at present cannot fully support the performance of advanced endosurgical procedures. They lack the dexterity required in making basic maneuvers, such as in the triangulation of surgical tools and in tissue manipulation, incision, and excision. Transmission of force from operator to point of action is suboptimal due to instability of the flexible endoscope. Moreover, the visual field is fixed by the direction of the endoscope, making visualization difficult when the field is constantly reoriented at every tilt of the tip of the endoscope. The major concern is the antiquated design of the semi-rigid endoscopy system, which has its lighting and accessory channels fixed in parallel to the endoscope, limiting 
the stability of field illumination and disabling off-axis maneuvering of endoscopically deployed surgical instruments.

\section{TECHNOLOGY SOLUTIONS FOR ADVANCED ENDOSCOPY}

Numerous innovative endoscopic systems have been developed to support advanced therapeutic endoscopy. Although not exclusively developed for colonoscopy, these new platforms or systems promise easier and safer colonoscopy with minimal discomfort to patients. Some of them have been approved by the US Food and Drug Administration (FDA), and/or have obtained the European Economic Area Conformité Européene (CE) mark certification. These technically enhanced and significantly improved novel systems include intuitively operated robotic endoscopic platforms/systems, mechanically enhanced and/or robotics-assisted endoscopes, and autonomously driven endoscopic locomotion devices that can effortlessly traverse the colon. Most robotic endoscopy platforms come with a computing system with dedicated software, an operator console, and a flexible endoscope with at least two multi-articulating operating arms capable of performing common surgical tasks such as triangulation of instruments, tissue manipulation (grasping, traction, retraction), approximation, cutting (incision, resection), closure of defect (suturing, clipping), and application of hemostasis in case of bleeding. In most cases, the optical system (light and camera) is also dissociated from the main instrument, and thus, separating the illumination of surgical field from endoscopic motion in order to provide an uninterrupted field of vision. Where tactile feedback is incorporated, the operator's perception of touch is enabled to facilitate appropriate application of force during the procedure. The advantage in incorporating intelligent robotic control is that as the machine takes over part of the work, the operator can be freed from tedious repetitive manual tasks and can focus on accurately performing the procedure.

\section{Robotic master and slave systems}

\section{The Master and Slave TransEndoluminal Robot}

The Master and Slave TransEndoluminal Robot (MASTER) (EndoMaster Pte Ltd., Singapore) is a multitasking flexible robotic endoscopic platform that uses an electromechanically controlled cable actuation system to drive the system. ${ }^{6}$ The platform consists of a master console, a telesurgical workstation, and a custom-designed therapeutic endoscope with two instrument channels (Fig. 1). Various end effectors (e.g., grasper, hook, cutter, needle, and electrocauterizers) can be deployed at the tip of the endoscope, with seven degrees of freedom (Fig. 2). The control of the robotic arms is intuitive, making it easy for the operator to use. The MASTER provides enhanced dexterity in surgical maneuvers, stable position-

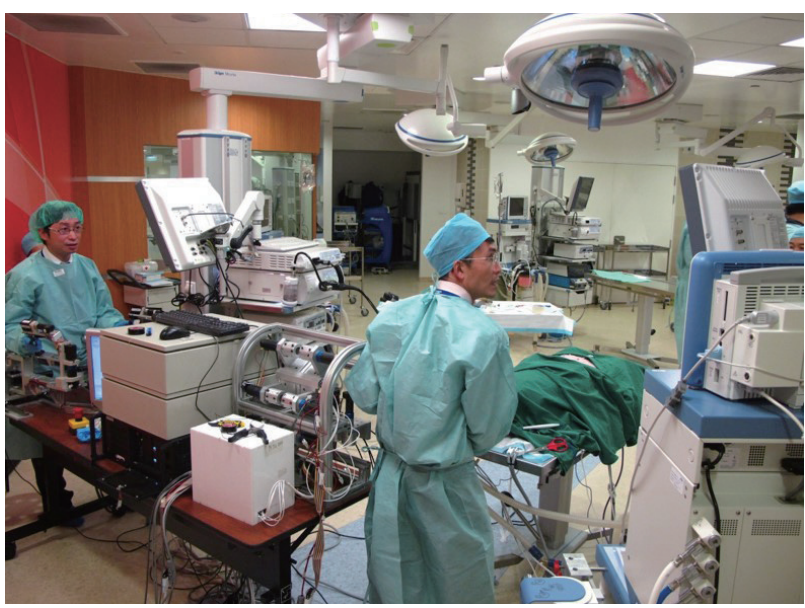

Fig. 1. Set-up of the entire Master and Slave TransEndoluminal Robot system, with the surgeon operating the master console of the robot at the left, and the endoscopist controlling the endoscope at the right.

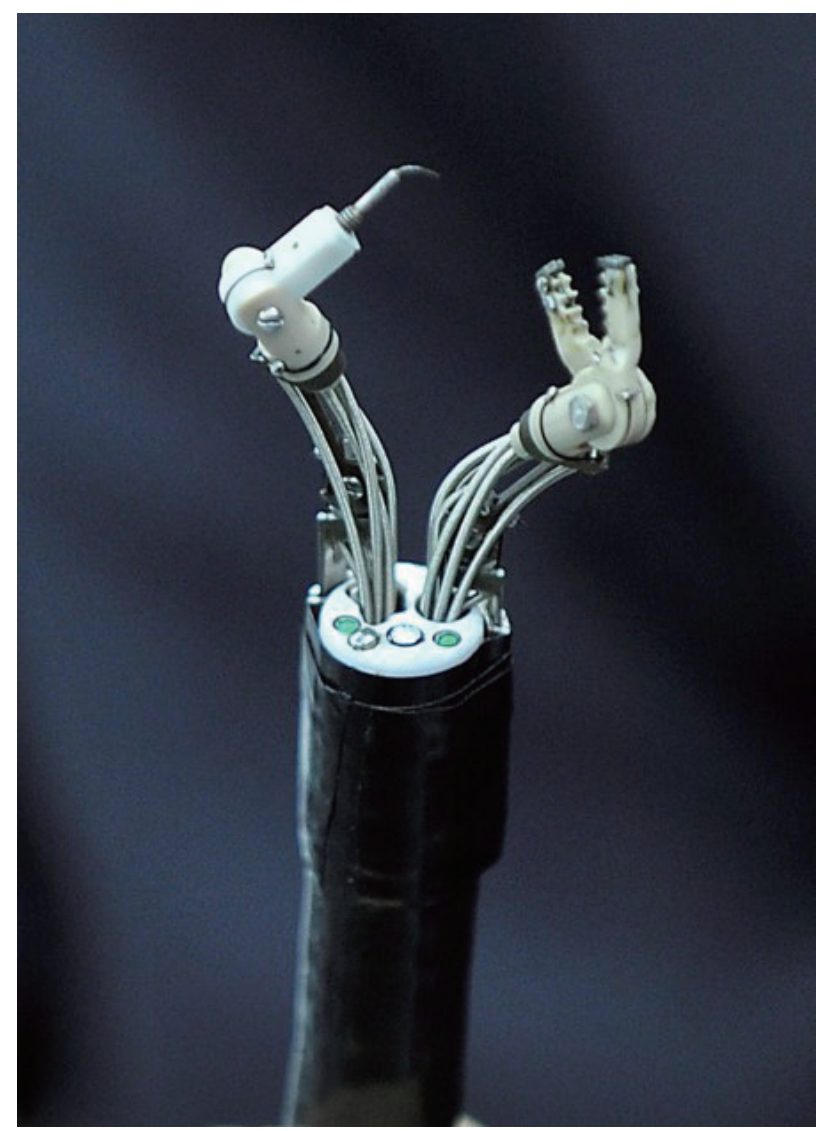

Fig. 2. The two effector arms of the Master and Slave TransEndoluminal Robot where different endosurgical instruments can be deployed. Each arm has seven degrees of freedom for spatial mobility of the instruments 
ing and efficient force transmission, and full visualization of the surgical field. Designed to handle both intraluminal and transluminal endoscopic procedures, ${ }^{7,8}$ the MASTER has been clinically validated for endoscopic sub-mucosal dissection of early gastric neoplasia, with an operation time comparable to that required using conventional methods. Its potential in applications such as transluminal gastric full thickness resection and liver wedge resection has been demonstrated in animal trials. ${ }^{10,11}$ Further development is ongoing to improve position control and introduce haptic capability.

\section{The single access and transluminal robotic assistant for sur- geons-ANUBIScope ${ }^{\mathrm{TM}}$}

The ANUBIScope (AnubiScope ${ }^{\mathrm{TM}}$; Karl Storz Endoskope, Tuttlingen, Germany) is a novel flexible therapeutic endoscopy platform that comes with multiple modules to support both endoluminal and extraluminal interventions of gastrointestinal lesions. The main endoscopic system features an endoscope with two expandable working channels for instrument deployment, and a third central channel for suction. Mounted at the distal tip of the endoscope are two opposing arms that provide two-way articulation, enabling easy triangulation of surgical instruments. The system can be operated manually as a standalone endoscope or robotically, through an intuitively designed haptic device. A single access and transluminal robotic assistant for surgeons provides further support for the performance of endoscopic transluminal procedures. The use of the AnubiScope ${ }^{\mathrm{TM}}$ in colorectal endoscopic submucosal dissection has been successfully demonstrated in animals. It has been shown to be superior to conventional endoscopes in terms of operation time and occurrence of adverse events. ${ }^{12}$

\section{Scorpion-shaped endoscopic robot by Kyushu University}

The scorpion-shaped endoscopic robot (Kyushu University, Fukuoka, Japan) is a relatively new concept of flexible endoscopy system built to meet the high technical demands of natural orifice transluminal endoscopic surgery. The prototype flexible endoscopic system has two cable-driven robotic arms and an integrated camera at the distal end of the endoscope. ${ }^{13}$ Each robotic arm is capable of vertical and horizontal movement, as well as movement to open/close the end-effectors. Magnetic sensors are installed to detect the exact location of the tip of the endoscope. It has haptic feedback capability to allow the operator to feel the touch as it manipulates the tissue. However, the system is still at an early stage of development.

\section{The ViaCath system}

The ViaCath system (Hansen Medical Inc., Mountain View, CA, USA) is a teleoperated endoscopic robot consisting of a mas- ter console and a slave drive system with an instrument channel fixed alongside the endoscope via an overtube ${ }^{14,15}$ It is adaptable to use with conventional endoscopes. The master console and the slave manipulators have a haptic interface, with seven degrees of freedom. The master slave system is cable actuated. The design enables infinite configurations of the flex section of the manipulator. Force generation is maximized by virtue of the stiff flex section. The system comes with a dedicated range of interchangeable electronically controlled end effectors, which include a grasper, electrocautery knife, scissors, and a needle holder. Designed for natural orifice transluminal surgery, it facilitates instrumental dexterity and procedural precision. Pre-clinical trials on porcine models have validated its use in endoscopic mucosal resection. ${ }^{14}$

\section{Robotic colonoscopic manipulation system by Yonsei Uni- versity}

This prototype robotic colonoscopic manipulation system was developed by Yonsei University College of Medicine, Seoul and Hanyang University, Ansan, Korea. It has a slave robot and an ergonomically designed master controller that controls the movements of the endoscopic slave manipulators. The master robot is equipped with a tilting and an insertion/ rotation device. The system is built with kinesthetic and haptic algorithm, and it does not require direct contact of the operator with the endoscope. ${ }^{16}$ The slave robot has a range of motion same as that of the flexible endoscope. Four degrees of freedom at the tip-end of the endoscope allow motions for its insertion, rotation, and tilting (maximum rotation angle: $360^{\circ}$; maximum tilting angles: $+\left(-180^{\circ}\right)$. The system can be adapted to a standard colonoscope by mounting it on the slave robot. A previous study showed that an expert endoscopist could complete the insertion of this robotic endoscope within 15 to 20 minutes, with $100 \%$ success rate. ${ }^{16}$ Development of this system, however, is ongoing.

\section{Auris Robotic Endoscopy System}

The Auris Robotic Endoscopy System (ARES) (Auris Health, Inc., Redwood City, CA, USA) is a teleoperated robotic flexible endoscopy system that features a pair of articulating arms with a long tube attached, allowing an endoscopist to steer a camera and other surgical implements inside the body of an individual through natural orifices. It has an intuitive instrument control interface, with an optional video-game type hand-held console for the operator to navigate micro-instruments deployed through a flexible endoscope. The system is aimed for use in natural orifice transluminal surgery. The first commercial ARES product, branded as Monarch Platform, has been cleared by FDA for use in bronchoscopic procedures. ARES is a streamlined and extensible platform that, with further customizations, can be used in other intraluminal and 
transluminal endoscopic applications such as those in colonoscopy. Development of this system, too, is ongoing.

\section{Robotics-driven autonomous locomotion systems}

\section{Medrobotics Flex ${ }^{\circledR}$ Robotic System}

Endoscopic robots that move like a snake are multi-articulated and are capable of autonomous flexible locomotion in the gastrointestinal tract. Prototypes of several snake-like endoscopic robots, including the well-known i-Snake ${ }^{\circledR}$, have been developed in the past decade. ${ }^{17-19}$ While most of them are either still under development or have been abandoned, the Flex ${ }^{\circledR}$ Robotic System (Medrobotics Corporation, Raynham, MA, USA) has been cleared by FDA for use in colonoscopy. The Flex ${ }^{\circledR}$ Robotic Colorectal System is made up of the Flex ${ }^{\circledR}$ Robotic System and Flex ${ }^{\circledR}$ Colorectal Drive. The colorectal drive is a steerable and shapeable robotic endoscope made up of multiple segments, with only one point of articulation between segments. The endoscope, with accessory channels, can autonomously and non-linearly be steered to the target site. Once in place, the endoscope can be stabilized and kept rigid to allow steady deployment of compatible endoscopic instruments such as graspers and needle drivers. This flexible robotic system is well-suited for local excision of neoplastic rectal, and potentially, sigmoid lesions. A trial involving a transanal total mesorectal excision in a cadaveric model has demonstrated the feasibility of the use of this system in resection and closure of trans-anal tissue. ${ }^{20}$

\section{The NeoGuide Endoscopy System}

The NeoGuide Endoscopy System (Intuitive Surgical, Sunnyvale, CA, USA) is a computer-aided colonoscope that consists of a 16-segment insertion tube that controls movements of the endoscope. The segments are independent and are electromechanically controlled to follow the contours of the colon. The leading segment has a position sensor that can sense the position of the endoscope tip, and another sensor that measures the depth of insertion. Computerized mapping enables the segments to crawl through the tortuous colon like a snake. Real-time 3D mapping of the colon enables identification of loops and live view of the position of the scope. By preventing the formation of colonic loops, the patient can be spared the unnecessary pain during colonoscopy. The system has been evaluated in a preliminary clinical trial and has shown high success in cecal intubation. $^{21}$

\section{The magnetically driven robotic platform}

An innovative magnetic-driven soft-tethered endoluminal robot has been developed by The BioRobotics Institute, Pisa, Italy. The endoluminal robot comprises of a workstation, a soft-tethered stereoscopic capsule with a magnet embedded, and a robotic guidance system, which includes a robotic arm, a dedicated setting module, an integrated localization, and teleoperated control modality. The smart stereoscopic capsule is magnetically actuated and is navigated with high reliability by an external magnet fixed on the robotic arm. The system is easier to navigate than the standard colonoscope is; it obviates the pain that typically accompanies excessive pushing, looping of the colonoscope, and inadvertent injury to the colon.

\section{The Endotics ${ }^{\circledR}$ System}

The Endotics ${ }^{\circledR}$ System (ERA Endoscopy, Peccioli, Italy) is a robotic-driven self-propelled disposable colonoscope. Considered a metamorphosis in robotic locomotion, it consists of a workstation and a sterile, disposable worm-shaped probe. It has a soft, flexible body, a head, a steerable tip, and an electro-pneumatic connector. The head contains LED lights and a camera with a 110-degree viewing angle. The workstation allows the endoscopist to control the disposable probe using a hand-held console. The probe can move semi-autonomously by means of clamps located at the proximal and distal ends of the probe. Inchworm-like manner of locomotion is achieved by repeated attach and release from the colorectal mucosa. For locomotion, the operator can steer the head of the probe to $180^{\circ}$ in every direction. The operator can also switch on/off functions such as rinsing, insufflation, and suction. In a study conducted on subjects with clinical or familial risk of colonic polyps/carcinomas, compared to the gold standard, the system was shown to have a sensitivity of $93.3 \%$ and a specificity of $100 \%$ for visualization of polyps. ${ }^{23}$ Studies on detection of colorectal polyps also showed superiority in pain reduction when this system was used, compared with conventional colonoscopy. The Endotics ${ }^{\circledR}$ System is CE-marked and is commercially available.

\section{Aer-O-Scope ${ }^{\mathrm{TM}}$ Colonoscopy System}

The Aer-O-Scope ${ }^{\mathrm{TM}}$ Colonoscope System (GI View Ltd., Ramat Gan, Israel) is a self-propelled disposable colonoscope with a circumferential omni-directional $360^{\circ}$ panoramic visualization. It comprises of a workstation with intuitive joystick control and a disposable colonoscope and scanner. The colonoscope is a $5.5-\mathrm{mm}$ soft multi-lumen tube with a unique pneumatic self-propulsion mechanism that uses balloons and low-pressure carbon dioxide gas for self-propelled intubation and suction. A pre-clinical study in a live swine model showed high detection rates for simulated colonic lesions. ${ }^{24} \mathrm{~A}$ small study of 56 subjects showed that the system has a cecal intubation and polyp detection rate of $98.2 \%$ and $87.5 \%$, respectively. There was no incidence of mucosal damage or adverse event. ${ }^{25}$ The Aer-O-Scope ${ }^{\mathrm{TM}}$ Colonoscope System has been cleared by 
the FDA for use in screening for colorectal cancer.

\section{Robotics-assisted technically enhanced systems}

\section{Robotic-assisted flexible endoscope system by Kyushu Uni- versity}

The robotic-assisted flexible endoscope (RAFE) has been developed by Kyushu University, Japan. The prototype system consists of a master controller, a motorized arm, and an endoscope holder. It is designed such that the endoscopist can control both the endoscope dials and the axis of the endoscope with a single hand. The endoscope grip is set into the endoscope holder, which houses two motorized wheels that drive the angle dials and valve buttons for suction, air supply, and water supply. The axial insertion-retraction of the endoscope is accomplished by the motorized arm. The coupling of the arm to the flexible part of an endoscope is free for axial rotation but fixed for axial insertion-retraction. The master controller is one-handle type, the movement of which controls the insertion-retraction, rotation, up-down, and rightleft movement of the endoscope. For operation, the robotic system can be adapted to a conventional endoscope. The operability of the RAFE has been demonstrated in endoscopic submucosal dissection in ex vivo porcine stomachs. ${ }^{26}$ En bloc resection was easily achieved by an expert endoscopist, while a non-endoscopist could control the endoscope more easily than he could control a manual endoscope. Further development of the RAFE is ongoing.

\section{The invendoscope}

The invendoscope SC210 (invendo medical GmbH, Kissing, Germany) is a revolutionary robotically assisted single-use colonoscope. This state-of-the-art disposable colonoscope is ergonomically designed with a reusable hand-held control unit used to activate all the endoscopic and software functions, and a disposable endoscopy sheath. Insertion and withdrawal of the colonoscope are controlled by the control unit. An endoscopic driving unit with eight drive wheels propels the inner sheath of the endoscope forward or backward. The colonoscope has a unique robotically driven tip armed with three white LEDs and a complementary metal-oxide-semiconductor chip. The tip can be flexed electro-hydraulically to $180^{\circ}$ in any direction and can move in circles, providing the operator with a complete view of the cavity. It also allows full retroflection for inspection of the mucosa behind colonic folds. A 3.2-mm working channel allows deployment of standard endoscopic instruments. Suction, insufflation, rinsing, and flushing can also be performed. The invendoscope generates its own internal force and requires minimal external pushing. A recent clinical study showed a cecal intubation rate of $98.4 \%$ (median time, $15 \mathrm{~min}$ ), without any pain, in $92 \%$ of patients. ${ }^{27}$ Polypectomies were successfully performed in 23 patients. The invendoscope SC210 is now commercially available in the US and Europe.

\section{The Endomina system}

The Endomina (Endo Tools Therapeutics, Nivelles, Belgium) is a universal triangulation platform that can be used to add triangulation capability to the conventional endoscope. It allows the endoscopist to use multiple tools and make complex maneuvers. The Endomina is actuated electromechanically through traction cables and controlled via two joysticks. It supports two operating channels, including one accommodating a therapeutic arm that moves with three degrees of freedom independently from the endoscope. The Endomina is designed for complex intraluminal and extraluminal interventional procedures. The performance of the system has been evaluated in complex tissue resection and apposition in various models. In a recent study on the performance of endoscopic sleeve gastroplasty involving transmural endoscopic sutures, the median procedure time while using this system was 126 (range, 75-200) minutes. No major intraoperative adverse event was reported. ${ }^{28}$ The clinical trial found the system safe and effective. The system is CE certified. As a universal triangulation system, it can potentially be used for other gastrointestinal interventions such as those in colonoscopy.

\section{Robotic steering and automated lumen centralization sys- tem}

The robotic steering and automated lumen centralization system (University of Twente, Enschede, Netherlands) is a prototype robotic platform using a new algorithm based on dark region centralization for colonoscopy steering. ${ }^{29}$ The system allows a conventional endoscope to be docked to its drive to take advantage of the system's capability. Control of the system is achieved via a joystick device. Movements of the endoscope are motorized and controlled via an intuitive interface. The tip of the endoscope may be driven remotely by the operator or through the image-based navigation algorithm. The operability of the system has been evaluated in a validated colon model, which showed that the system enabled novices, but not experienced endoscopists, to reach the cecum faster during colonoscopy. ${ }^{30}$ Moreover, the novices detected more polyps when this system was used. The median cecal intubation time required by the system was $781 \mathrm{~s}$, compared with $129 \mathrm{~s}$ taken by a manually steered conventional endoscope.

\section{CONCLUSIONS}

Recent technological advancements in endoscopic instru- 
mentation have overcome multiple technical barriers in performing interventional therapeutic endoscopy. With uninterrupted visualization of the surgical field, better system control, easier deployment of instruments, enhanced dexterity and precision for surgical maneuvers, robotics-enabled systems allow intricate therapeutic procedures to be performed more easily. As robotic endoscopy is a developing field, the best in robotics-enabled endoscope innovations is probably yet to emerge; however, current innovations are already driving developments in endosurgical intervention techniques, including those applicable to colonoscopy.

\section{Conflicts of Interest}

Khek Yu Ho is the co-founder of EndoMaster Pte Ltd., Singapore. Other author has no financial conflicts of interest.

\section{REFERENCES}

1. ASGE Training Committee, Sedlack RE, Coyle WJ, et al. ASGE's assessment of competency in endoscopy evaluation tools for colonoscopy and EGD. Gastrointest Endosc 2014;79:1-7.

2. Fujishiro M, Yahagi N, Kakushima N, et al. Outcomes of endoscopic submucosal dissection for colorectal epithelial neoplasms in 200 consecutive cases. Clin Gastroenterol Hepatol 2007;5:678-683; quiz 645.

3. Saito Y, Fukuzawa M, Matsuda T, et al. Clinical outcome of endoscopic submucosal dissection versus endoscopic mucosal resection of large colorectal tumors as determined by curative resection. Surg Endosc 2010;24:343-352.

4. Tanaka S, Oka S, Kaneko I, et al. Endoscopic submucosal dissection for colorectal neoplasia: possibility of standardization. Gastrointest Endosc 2007;66:100-107.

5. Yoon JY, Kim JH, Lee JY, et al. Clinical outcomes for patients with perforations during endoscopic submucosal dissection of laterally spreading tumors of the colorectum. Surg Endosc 2013;27:487-493.

6. Phee SJ, Low SC, Huynh VA, Kencana AP, Sun ZL, Yang K. Master and slave transluminal endoscopic robot (MASTER) for natural orifice transluminal endoscopic surgery (NOTES). Conf Proc IEEE Eng Med Biol Soc 2009;2009:1192-1195.

7. Wang Z, Phee SJ, Lomanto D, et al. Endoscopic submucosal dissection of gastric lesions by using a master and slave transluminal endoscopic robot: an animal survival study. Endoscopy 2012;44:690-694.

8. Ho KY, Phee SJ, Shabbir A, et al. Endoscopic submucosal dissection of gastric lesions by using a master and slave transluminal endoscopic robot (MASTER). Gastrointest Endosc 2010;72:593-599.

9. Phee SJ, Reddy N, Chiu PW, et al. Robot-assisted endoscopic submucosal dissection is effective in treating patients with early-stage gastric neoplasia. Clin Gastroenterol Hepatol 2012;10:1117-1121.

10. Phee SJ, Ho KY, Lomanto D, et al. Natural orifice transgastric endoscopic wedge hepatic resection in an experimental model using an intuitively controlled master and slave transluminal endoscopic robot (MASTER). Surg Endosc 2010;24:2293-2298.

11. Chiu PW, Phee SJ, Wang Z, et al. Feasibility of full-thickness gastric resection using master and slave transluminal endoscopic robot and closure by Overstitch: a preclinical study. Surg Endosc 2014;28:319-324.
12. Diana M, Chung $\mathrm{H}$, Liu KH, et al. Endoluminal surgical triangulation: overcoming challenges of colonic endoscopic submucosal dissections using a novel flexible endoscopic surgical platform: feasibility study in a porcine model. Surg Endosc 2013;27:4130-4135.

13. Suzuki N, Hattori A, Tanoue K, et al. Scorpion shaped endoscopic surgical robot for NOTES and SPS with augmented reality functions. In: Medical Imaging and Augmented Reality - 5th International Workshop, MIAR 2010; 2010 Sep 19-20; Beijing, China. Berlin: Springer-Verlag Berlin Heidelberg; 2010. p. 541-550.

14. Rothstein RI, Ailinger RA, Peine W. Computer-assisted endoscopic robot system for advanced therapeutic procedures. Gastrointest Endosc 2004;59:P113.

15. Franzino RJ. The Laprotek surgical system and the next generation of robotics. Surg Clin North Am 2003;83:1317-1320.

16. Woo J, Choi JH, Seo JT, Kim TI, Yi BJ. Development of a robotic colonoscopic manipulation system, using haptic feedback algorithm. Yonsei Med J 2017;58:139-143.

17. Kwok KW, Tsoi KH, Vitiello V, et al. Dimensionality reduction in controlling articulated snake robot for endoscopy under dynamic active constraints. IEEE Trans Robot 2013;29:15-31.

18. Patel N, Seneci CA, Shang J, et al. Evaluation of a novel flexible snake robot for endoluminal surgery. Surg Endosc 2015;29:3349-3355.

19. Warren A, Mountney P, Noonan D, Yang GZ. Horizon stabilized--dynamic view expansion for robotic assisted surgery (HS-DVE). Int J Comput Assist Radiol Surg 2012;7:281-288.

20. Atallah S. Assessment of a flexible robotic system for endoluminal applications and transanal total mesorectal excision (taTME): could this be the solution we have been searching for? Tech Coloproctol 2017;21:809814.

21. Eickhoff A, van Dam J, Jakobs R, et al. Computer-assisted colonoscopy (the NeoGuide Endoscopy System): results of the first human clinical trial ("PACE study"). Am J Gastroenterol 2007;102:261-266.

22. Bianchi F, Ciuti G, Koulaouzidis A, et al. An innovative robotic platform for magnetically-driven painless colonoscopy. Ann Transl Med 2017;5:421.

23. Tumino E, Sacco R, Bertini M, Bertoni M, Parisi G, Capria A. Endotics system vs colonoscopy for the detection of polyps. World J Gastroenterol 2010;16:5452-5456.

24. Gluck N, Fishman S, Melhem A, Goldfarb S, Halpern Z, Santo E. A novel colonoscope with panoramic visualization detected more simulated polyps than conventional colonoscopy in a live swine model. Endosc Int Open 2015;3:E642-E645.

25. Gluck N, Melhem A, Halpern Z, Mergener K, Santo E. A novel self-propelled disposable colonoscope is effective for colonoscopy in humans (with video). Gastrointest Endosc 2016;83:998-1004.e1.

26. Iwasa T, Nakadate R, Onogi S, et al. A new robotic-assisted flexible endoscope with single-hand control: endoscopic submucosal dissection in the ex vivo porcine stomach. Surg Endosc 2018:32:3386-3392.

27. Rösch T, Adler A, Pohl H, et al. A motor-driven single-use colonoscope controlled with a hand-held device: a feasibility study in volunteers. Gastrointest Endosc 2008;67:1139-1146.

28. Huberty V, Ibrahim M, Hiernaux M, Chau A, Dugardeyn S, Deviere J. Safety and feasibility of an endoluminal-suturing device for endoscopic gastric reduction (with video). Gastrointest Endosc 2017;85:833-837.

29. van der Stap N, Rozeboom ED, Pullens HJ, van der Heijden F, Broeders IA. Feasibility of automated target centralization in colonoscopy. Int J Comput Assist Radiol Surg 2016;11:457-465.

30. Pullens HJ, van der Stap N, Rozeboom ED, et al. Colonoscopy with robotic steering and automated lumen centralization: a feasibility study in a colon model. Endoscopy 2016;48:286-290. 